\title{
A Micropower Vision Processor for Parallel Object Positioning and Sizing
}

\author{
Timothy G Constandinou and Chris Toumazou \\ The Institute of Biomedical Engineering, Imperial College London \\ South Kensington Campus, London, SW7 2AZ, United Kingdom \\ Email: t.constandinou@imperial.ac.uk, c.toumazou@imperial.ac.uk
}

\begin{abstract}
A hybrid vision chip is presented for real-time object-based processing for tasks such as positioning and sizing of enclosed objects. This system presents the first artificial silicon retina capable of position and size determination of multiple objects in true parallel fashion. Based on a novel distributed algorithm, this approach uses the input image to enclose a feedback loop to realise a data-driven pulsating action. The fabricated device is shown to achieve a computation-efficiency of at least 725 million instructions per second per milliwatt and capable of processing up to 2000 frames per second.
\end{abstract}

\section{INTRODUCTION}

Centroid detection and target tracking have been tasks traditionally associated with advanced military and space applications. However these same tasks are fundamental to the more generic field of image recognition. Traditional image processing techniques effectively perform low-level tasks such as conditioning and filtering but typically output a matrix of pixels, constituting an image. For perceptive vision applications it is paramount to cluster together pixels in a region of interest and provide a single entity. This task is often referred to as object segmentation. Having performed this, it is useful to define the object using a co-ordinate and magnitude to represent its centroid and size respectively. Having such highlevel information available can provide enhanced and added functionality to several applications. For example, in machine vision for autonomous navigation, automation of surveillance or security camera tasks, image stabilisation for medical instrumentation and biochemical cellular migration/population analysis.

Previous work has produced many centroiding vision chips, Eg. [1] [2] based on the centre-of-mass (COM) computation utilising two (i.e. row and column) one-dimensional summations at the side of the array. Other systems have combined this with additional functionality, for example, to combine centroiding with motion detection [3] [4] or embed an APS imager [5]. Multiple centroid capability has been achieved by using a window and search type algorithm to direct the COM computation [6] [7].

This paper reports the first vision chip capable of objectposition detecting and sizing of unlimited objects in genuine parallelism.

\section{Bio-Puls Ating Contour Reduction Algorithm}

This system directly implements the "Bio-pulsating Contour Reduction" algorithm [8], designed for object-based process-
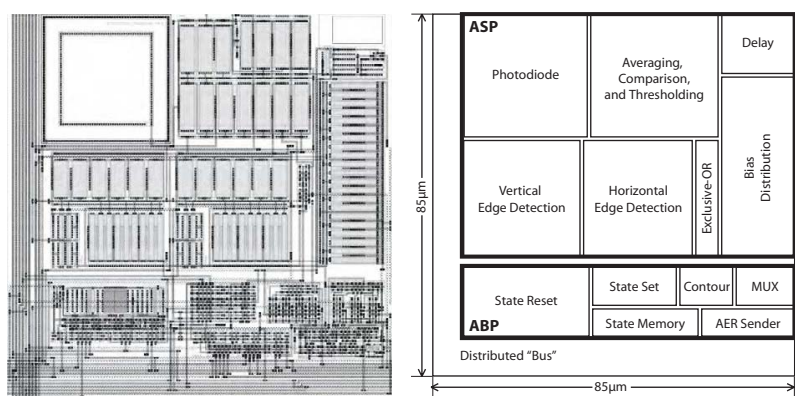

Fig. 1. The regular cell layout (top) and floorplan (bottom). The cell size is $85 \mu \mathrm{m} \times 85 \mu \mathrm{m}$ with $30 \mu \mathrm{m} \times 30 \mu \mathrm{m}$ active photodiode area, giving a $12.5 \%$ surface fill factor. Metal layers 5 and 6 have been excluded for clarity.

ing including positioning and sizing of simple objects. Circular blob-like objects with an intensity differing from the background level can be segmented and their size and position ("centroid") determined using a distributed binary algorithm. It is important to stress that the detected "centroid" is only an accurate centre-of-mass (COM) for regular, round objects, otherwise this represents an estimation to object position.

This uses an edge-detection technique to form the contours and trigger the data-driven processing. On detection of an object boundary, the initial state for the signal flow is set. By propagating an inward fill, the contour can be reduced until it converges to the "centre". The central point is detected by utilising spatiotemporal integration. On "centroid" detection, the object is reset and output transmitted, thus realising an inward pulsating action. Furthermore, the frequency of pulsation can be directly used to determine the object size.

\section{System ARChitecture AND Circuit IMPLEMENTATION}

The complete system architecture and circuit implementation is illustrated in Fig. 2. The architecture consists of three main components: the address-event communication hardware, the current distribution network and the distributed pixel processing array. The address-event communication implements a fully-arbitrated scheme adapted from [9] (circuits shown in Fig. 2c). The current distribution network is used to duplicate bias currents at close physical proximity and thus reduce mismatch error. The pixel implementation (layout and floorplan given in Fig. 1) consists of three main components: the photodiode $(30 \mu m \times 30 \mu m$ n-well/p-substrate 


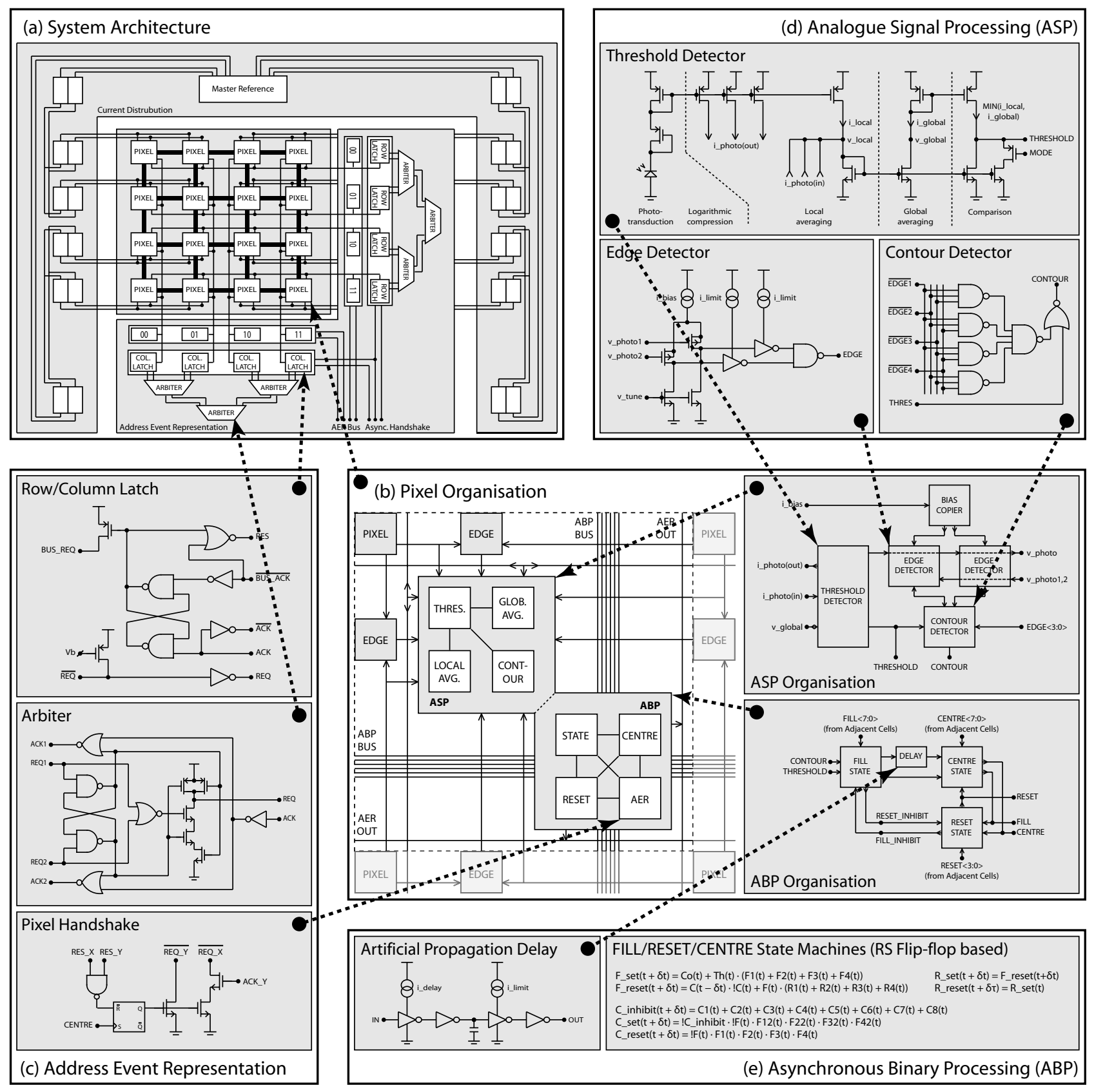

Fig. 2. Implementation details for the Micropower Object Positioning Vision Processor. Shown are: (a) General system architecture, (b) in-pixel organisation, (c) Address-Event Representation circuits, (d) Analogue Signal Processing circuits and (e) Asynchronous Binary Processing Circuits.

parasitic junction), Analogue Signal Processor (ASP) and Asynchronous Binary Processor (ABP).

\section{A. ASP Circuits (Fig. 2d)}

These circuits feature extract two binary signals per pixel (THRESHOLD and CONTOUR) from the input image.

1) Threshold Detection: Current-mode averaging and current comparator circuits are used to generate the THRESHOLD signal indicating whether a pixels intensity is below or above the global average.

2) Edge/Contour Detection: A comparator based on a current-starved differential pair [10] is used to edge detect between every two adjacent pixels. Subsequently, using EDGE signals from adjacent pixels, the CONTOUR signal is produced on determining a continuous edge.

\section{B. ABP Circuits (Fig. 2e)}

These circuits facilitate the distributed binary algorithm to "centroid" detect acting upon binary outputs of the ASP.

1) FILL state machine: This determines when a pixels fill status becomes asserted, facilitating the inward "fill". This is either due to the pixel lying on a object contour or to an inward propagation. 

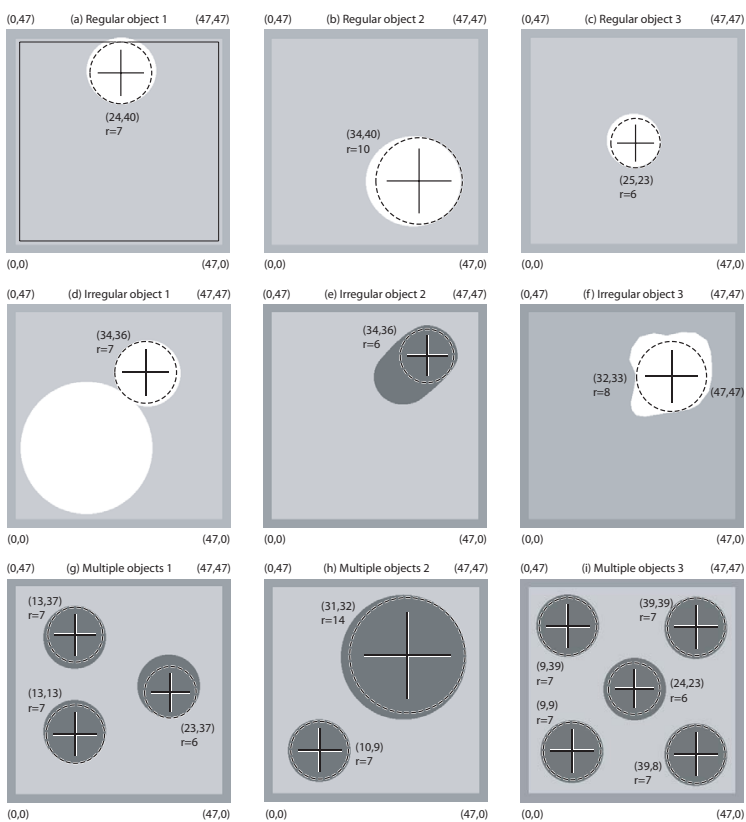

Fig. 3. Test images with pixel grid overlayed indicating measured centroid position and size. Included are: (a-c) Regular objects, (d-f) Irregular objects and (g-i) Multiple objects.

2) Artificial Delay: This limits the rate of inward propagation, i.e. an artificial delay is inserted before each pixels "Set FILL" logic. The delay is created by thresholding to capacitive charging of a bias current.

3) CENTROID state machine: This determines whether a CENTRE has been detected, by aggregating FILL inputs from surrounding cells.

4) RESET state machine: This determines when a pixels fill status becomes reset, facilitating the outward "unfill" or back-propagation. This is either due to the pixel detecting a CENTRE or to a back-propagation.

\section{FABRICATED SYSTEM}

\section{A. General Functionality}

A custom testboard setup (Microcontroller/PC-based AER readout) has been developed to confirm system functionality within the intended design specifications. Sample images, projected onto the chip and corresponding measurements are presented in Fig. 3. This illustrates both single and multiple object position and size determination. Typically the measured object position and size measurements are within the actual boundaries. Furthermore, uneven objects are successfully detected but with inaccurate centroid and position estimates, again within the actual object boundaries (see Fig. 3e,f). However, overlapping objects are detected as a single uneven object (Fig. 3d).

\section{B. Measured Results}

1) Accuracy: As expected this system is intrinsically limited to single pixel resolution. An interesting observation has been a small random deviation ( \pm 2 pixels) in object position, resulting in a similar deviation in object size. This is able (a)

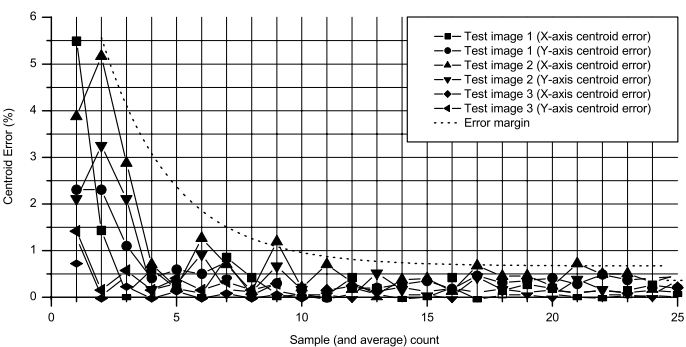

(b)

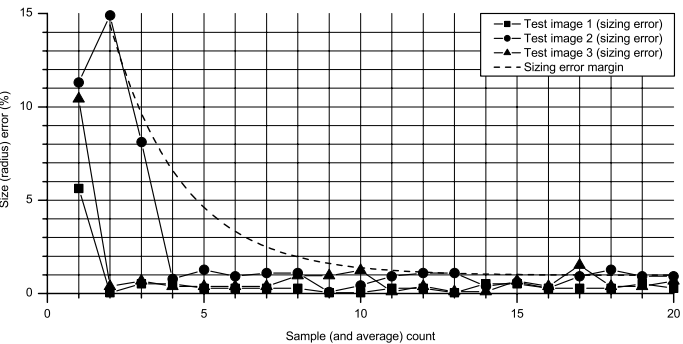

Fig. 4. Pseudo-dithering providing increased accuracy through successive averaging for object (a) position and (b) radius.

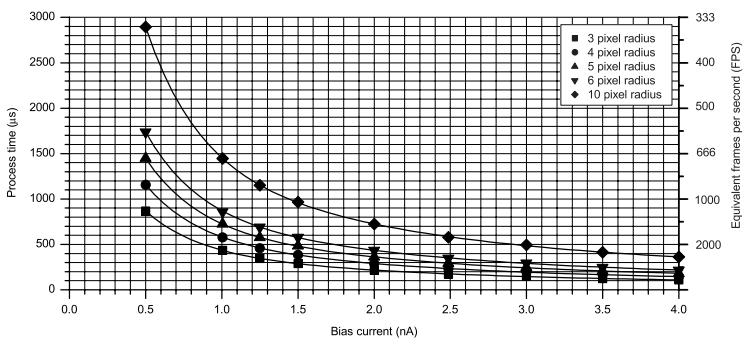

Fig. 5. Dependance of process time on bias current, for input images with maximum objects sizes of $3,4,5,6$ and 8 pixel radius.

to provide sub-pixel accuracy (through successive averaging) having a pseudo-dithering effect. This is explained due to an edge effect caused by an imperfectly focused image or a graded object boundary. Subsequently, the static (spatial) fixed-pattern noise (FPN) coupled with the (temporal) flicker noise within the edge detector blocks provide this statisticallybiased dithering effect. This results in a mechanism to enable processing time to be tradable with accuracy, illustrated by the trend shown in Fig. 4.

2) Processing Time: Although the asynchronous nature of this system produces temporally uncorrelated events between different objects (due to the local resetting), the algorithm can be run in a "single-shot" mode. This can be used by applying a clock to the global reset input, therefore realising a clocked or frame-based output. The limiting factor to processing speed is the maximum object size being analysed, i.e. contour reduction cycle. This can be tuned as the internal propagation delay is controlled by the bias current, illustrated in Fig. 5 .

3) Power Consumption: The measured power consumption is generally within $\pm 10 \%$ the simulated levels. The effect of illumination and bias current on power consumption is illustrated in Fig. 6. An unusual feature is that by decreasing bias current (below the $2.5 \mathrm{nA}$ nominal) it substantially increases power consumption. This is because the transconductance 
(a)

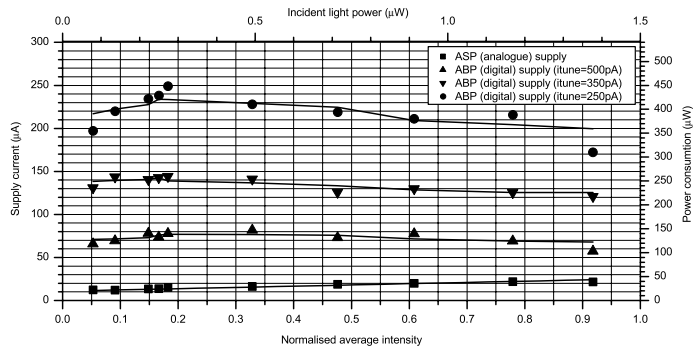

(b)

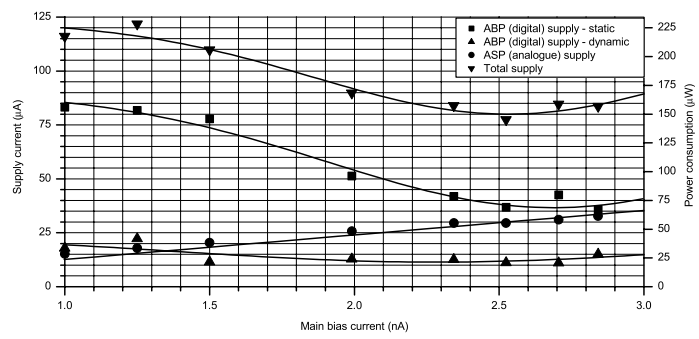

Fig. 6. Measured total system current consumption illustrating dependance on (a) illumination level and (b) edge detection bias current.

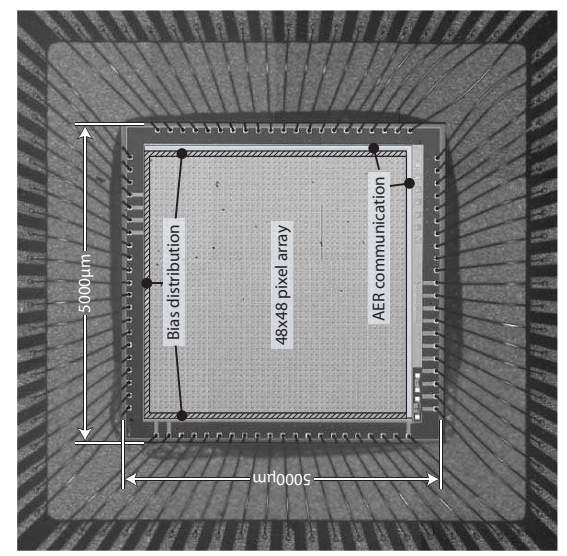

Fig. 7. Microphotograph of the Micropower Object Positioning Vision Processor.

within the edge comparators is reduced, causing the digital logic to operate with intermediate voltage inputs.

\section{CONCLUSION}

A vision processing chip has been presented for object position and size determination. It is the first system reporting parallel, multiple object (unlimited) processing capability. Furthermore, the developed system demonstrates high computational efficiency; implementing a computationally intensive algorithm with micropower consumption. The fabricated system (microphotograph shown in Fig. 7) has shown to utilise fixed pattern noise favourably; both reducing power consumption (through increased mismatch on edge detectors) and increased accuracy for moving objects (through dithering with successive sampling). The achieved system specification is summarised in Table. I.

\begin{tabular}{|c|c|}
\hline Technology & UMC $0.18 \mu m$ MM/RF CMOS \\
\hline Supply voltage & $1.8 \mathrm{~V}$ core $(3.3 \mathrm{~V} \mathrm{I} / \mathrm{O})$ \\
\hline \multirow[t]{3}{*}{ Bias current range } & $50 \mathrm{nA}$ to $2 \mu A$ (for $I_{a e r}$ ) \\
\hline & $250 \mathrm{pA}$ to $10 \mathrm{nA}$ (for $I_{\text {bias }}$ ) \\
\hline & $50 \mathrm{pA}$ to $2 \mathrm{nA}$ (for $\left.I_{\text {tune }}\right)$ \\
\hline Photosensitivity & $100 \mathrm{nW} / \mathrm{cm}^{2}$ to $100 \mathrm{~mW} / \mathrm{cm}^{2}$ \\
\hline Responsivity & $0.32 A / W c^{2}(\lambda=650 \mathrm{~nm})$ \\
\hline Pixel size & $85 \mu m \times 85 \mu m$ \\
\hline Surface fill factor & $12.46 \%$ \\
\hline Pixel device count & 277 \\
\hline Pixel power & $\dagger 96.48 \mathrm{nW}$ (total) \\
\hline Die dimensions & $5 \mathrm{~mm} \times 5 \mathrm{~mm}$ \\
\hline Array size & $48 \times 48$ pixels \\
\hline System device count & 745,200 \\
\hline System power & $\dagger 243.6 \mu W$ (total) \\
\hline Accuracy (centroid and radius) & \pm 1 pixel \\
\hline Equivalent image process time & $0.5 \mathrm{~ms}$ \\
\hline Address-event bandwidth & $0.61 \mathrm{MHz}\left(\right.$ at $\left.I_{a e r}=1 \mu A\right)$ \\
\hline Equivalent computational efficiency & $\dagger 1.38 \mu W$ per MIPS \\
\hline
\end{tabular}

$\dagger$ For $\mathrm{n}=5, \mathrm{r}=10, I_{\text {photo } A v}=6 \mu \mathrm{W} / \mathrm{cm}^{2}, I_{\text {bias }}=2.5 \mathrm{nA}, I_{\text {tune }}=250 \mathrm{pA}$

TABLE I

SYSTEM PROPERTIES AND PERFORMANCE SUMMARY

\section{ACKNOWLEDGMENT}

The authors would like to acknowledge the Basic Technology grant (UKRC GR/R87642/02) and the AMx technology grant (EPSRC GR/R96583/01), in addition to Toumaz Technology Limited for supporting this research. The authors would also like to thank Tor Sverre Lande and Julius Georgiou for many useful discussions and Philipp Häfliger for providing us with access to his address-event design libraries.

\section{REFERENCES}

[1] N. Massari, L. Gonzo, M. Gottardi and A. Simoni, "A Fast CMOS Optical Position Sensor with High subpixel Resolution," IEEE Trans. on Instr. Meas., vol. 53, no. 1, pp. 116-123, 2004.

[2] B. H. Pio et al, "Integration of a Photodiode Array \& Centroid Processing on a single CMOS Chip for a Real-time Shack-Hartmann Wavefront Sensor," IEEE Sensors J., vol. 4, no. 6, pp. 787-794, 2004.

[3] R. Etienne-Cummings, V. Gruev and M. Abdel-Ghani, "VLSI Implementation of Motion Centroid Localization for Autonomous Navigation," Advances in Neural Information Processing Systems, vol. 10, pp. 685-691, 1998.

[4] G. Indiveri, "Neuromorphic analog VLSI sensor for visual tracking," IEEE Trans. Circuits Syst. II, vol. 46, no. 11, pp. 1337-1347, 1999.

[5] M. A. Clapp and R. Etienne-Cummings, "A Dual Pixel-type Array for Imaging and Motion Centroin Localization," IEEE Sensors J., vol. 2, no. 6, pp. 529-548, 2002.

[6] J. Akita, A. Watanabe, O. Tooyama, M. Miyama, M. Yoshimoto, "An Image Sensor with Fast Objects' Position Extraction Function,” IEEE Trans. Electron Devices, vol. 50, no. 1, pp. 184-190, 2003.

[7] T. Komuro, I. Ishii, M. Ishikawa and A. Yoshida, "A Digital Vision Chip Specialized for High-Speed Target Tracking," IEEE Trans. Electron Devices, vol. 50, no. 1, pp. 191-199, 2003.

[8] T. G. Constandinou, T. S. Lande and C. Toumazou, "Bio-pulsating architecture for object-based processing in next generation vision systems," IEE Elec. Lett., vol. 30, no. 16, pp. 1169-1170, 2003.

[9] P. Häfliger, A Spike-based Learning Rule and its Implementation in Analog Hardware”. PhD thesis, ETH Zürich, Switzerland, 2000.

[10] T. G. Constandinou, J. Georgiou and C. Toumazou, "A Nanopower Tuneable Edge Detection Circuit," Proc. IEEE Int. Symp. on Circuits Syst., vol. 1, pp. 449-452, 2004 